Georgia Hardavella1 ${ }^{1}$, Armin Frille ${ }^{2,3}$, Christina Theochari', Elli Keramida ${ }^{1}$, Elena Bellou', Andreas Fotineas ${ }^{4}$, Irma Bracka', Loukia Pappa', Vaia Zagana ${ }^{5}$, Maria Palamiotou ${ }^{5}$, Panagiotis Demertzis ${ }^{1}$, loannis Karampinis ${ }^{6}$

georgiahardavella@hotmail.com

Review

\title{
Multidisciplinary care models for patients with lung cancer
}

Multidisciplinary care is the cornerstone of lung cancer treatment in the developed world, even though there is a relative lack of consistent evidence that this care model improves outcomes. In this review, we present the available literature regarding how to set up and run an efficient multidisciplinary care model for lung cancer patients with emphasis on team members' roles and responsibilities. Moreover, we present some limited evidence about multidisciplinary care and its impact on lung cancer outcomes and survival.

This review provides simple guidance on setting up and running a multidisciplinary service for lung cancer patients. It highlights the importance of defined roles and responsibilities for team members. It also presents concise information based on the literature regarding the impact of multidisciplinary care in lung cancer outcomes (e.g. survival of patients undergoing lung cancer surgery).

\section{Introduction}

Lung cancer care has a complicated nature and mandates a unique level of care coordination where time is a perishable resource. The "Framework for action on interprofessional education and collaborative practice" [1], published by the World Health Organization (WHO), reports that effective interprofessional/multidisciplinary teamwork is considered an essential component for the delivery of high-quality patient care in an increasingly complex medical environment and multidisciplinary collaboration plays an important role in improving healthcare services and patient outcomes.

Multidisciplinary care has emerged as a significant approach in cancer management and it has been the cornerstone of the diagnosis and treatment pathways of lung cancer patients [2, 3].

Following the Lisbon roundtable held during the Portuguese European Union Presidency in 2007 [4], a multidisciplinary team (MDT) approach to lung cancer care is a requirement for lung cancer centres in Europe, this is also the case in Australia and the USA [2, 4, 5]. MDT meetings are also known as "tumour boards", "MDT case reviews" or "MDT cancer conferences" in different healthcare systems. These terms may represent differences in standard operating procedures, organisational structure and decision-making processes; however, their focus is to facilitate a dialogue and subsequent ongoing collaboration between healthcare professionals with complementary

@ERSpublications

Despite lack of robust evidence, multidisciplinary care remains the cornerstone in lung cancer care https://bit.ly/3qFXy2w

Cite as: Hardavella $G$ Frille A, Theochari C, et al. Multidisciplinary care models for patients with lung cancer. Breathe 2020; 16: 200076. 
roles and areas of expertise in order to reach a consensus on evidence-based management plans tailored to the patients' needs [3].

\section{Lung cancer care models}

Lung cancer care models can vary among different countries or even among institutions within the same country depending on the infrastructure, available resources, quality standards and operating procedures [6]. Three main forms can be identified depending on whether the multidisciplinary involvement is continuous throughout the patient pathway or whether it is fragmented or "on demand" [7].

\section{1) Serial referral system}

This system involves individual specialist referrals offered as the patient progresses in the diagnostic or treatment pathway [7]. This is a "refer-as-you-go" system that is shaped by demand. The serial referral system may prolong the patient's pathway as every specialist referral comes with its own separate waiting times for each outpatient appointment and subsequently there are significant delays in the patient pathway and treatment [8]. In this system, medics work in isolation rather than in partnership and this may lead to inappropriate choice of treatment options as specialists tend to offer options that are familiar to them or are more easily accessible rather than options tailored to the patient's needs $[8,9]$. Allied health professionals (AHP) do not seem to be directly involved in the patient pathway unless the medics are working in a team where an AHP is available.

\section{2) MDT meeting (tumour board) focused model}

Lung cancer MDT (tumour board) meetings are hubs offering integrated multidisciplinary lung cancer care tailored to the patient's needs. They involve the continuous collaboration of lung cancer specialists throughout the patient continuum where patient cases are presented and discussed for a diagnostic or treatment plan to be put in place [7].

The quality of the presentation heavily affects the quality of the diagnostic/therapeutic recommendations made by the team; therefore, it is vital that cases are presented by a healthcare professional that is familiar with the patient's continuum $[7,10]$.

\section{3) MDT clinic-based model}

This model includes a dedicated centralised cancer clinic space with ample clinic rooms and resources to allow a sequence of patient interactions with various lung cancer specialists depending on the patient's position on the pathway [7, 9].

Lung cancer care involves multimodality staging and treatment; therefore, it is crucial to host all healthcare professionals involved in one place at the same time as this is associated with improved patient experience [9]. Patients are offered the opportunity to have specialist consultations within a single clinic where they can communicate about their diagnosis and treatment. This process is important, as it offers patients easy access to all lung cancer specialists but also ensures the continuity of their care $[11,12]$.

An American Thoracic Society (ATS)/European Respiratory Society (ERS) statement has recommended that all centres offering thoracic oncology services should have multidisciplinary clinics and a thoracic oncology MDT meeting (models 1 and 3) [13]. Based on our experience, this model offers optimal integrated multidisciplinary lung cancer care. It provides a forum for seamless collaboration between all members of the lung cancer team and ensures close communication with patients to ensure optimal treatment plans.

\section{The challenge of setting up a functional multidisciplinary lung cancer service.}

Organising a functional multidisciplinary lung cancer service is a challenging, yet rewarding process. An important aspect of multidisciplinary lung cancer care is an emphasis on patient-focused care and an attempt to improve the patient journey through collaboration, communication, and streamlining of diagnostics and treatment (figure 1).

Active recruitment of interested physicians can be achieved by networking and socialising amongst members of the hospital/community healthcare team with relevant expertise. The assembled team needs to commit to attending regular meetings and to referring cancer patients that could benefit from multidisciplinary discussion [14].

Table 1 summarises the anticipated challenges. Inappropriate job planning and time management can compromise clinician's MDT attendance significantly. On some occasions, MDT members may get absorbed by daily clinical ward duties and may find it difficult to attend all or most of the MDT meetings (tumour board). To avoid this, all MDT members should have dedicated and protected time to attend the MDT (tumour board) meeting and subsequent MDT clinic and these activities should be planned appropriately. Their job plan should also include some additional time to cover administrative duties that may arise from the MDT meeting $[1,15]$. Poor MDT attendance is accompanied by insufficient preparation and inadequate clinical information, unequal contributions to MDT discussions and overseeing of patient-centred information. This 


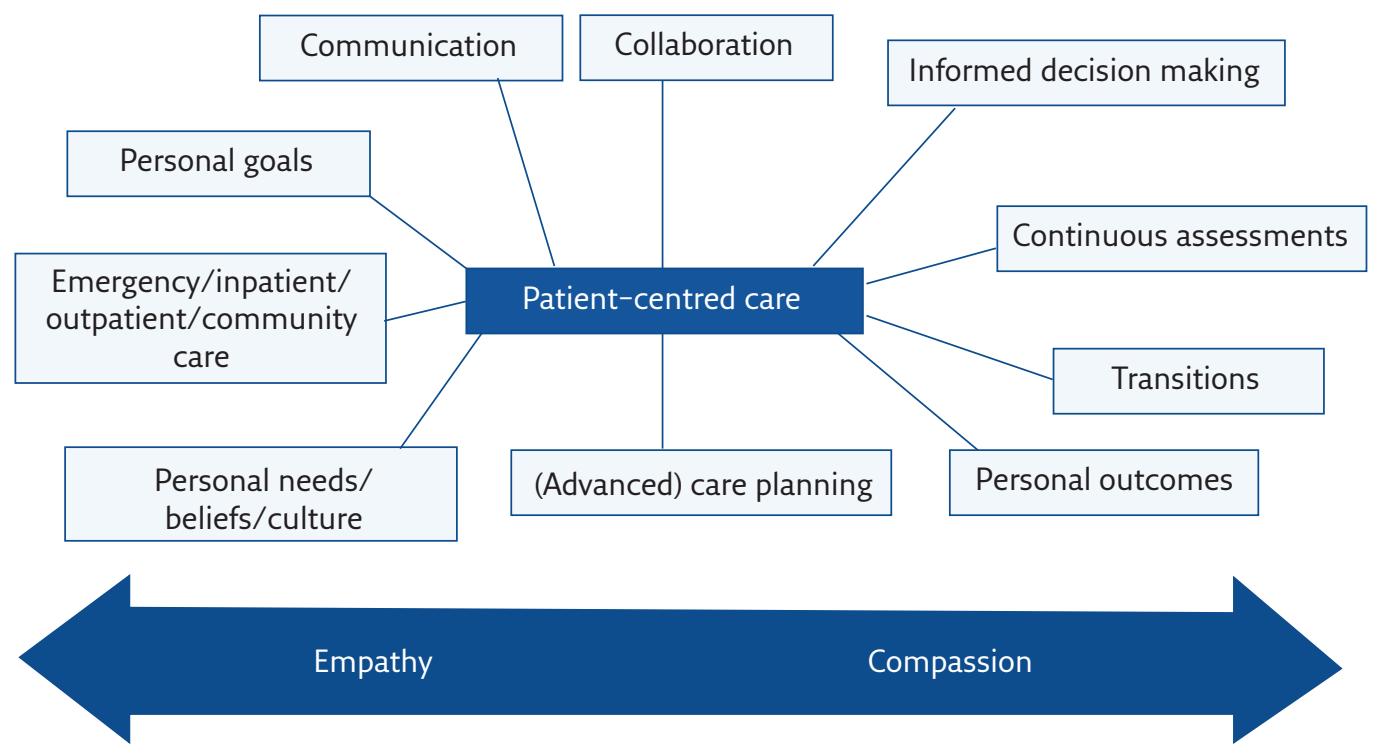

Figure 1 Achieving patient-centred care in a multidisciplinary setting.

series of events creates a vicious circle and impacts on the quality of MDT discussions [16, 17].

Identifying a competent MDT lead clinician is a prerequisite for MDT (tumour board) efficiency. The MDT lead/chair is an established physician leader in the hospital and can be from any specialty participating in the lung cancer MDT meeting. Leadership is critical to ensure diversity and equal expression of all opinions from different specialties, to support openness and blame-free culture, reinforce inclusivity, ensure the meeting is on track and audit outcomes to optimise performance [3, 18]. Poor leadership, insufficient teamwork and time pressure are barriers for efficient MDT working $[19,20]$. The MDT meeting lead needs to ensure accessible record keeping, documentation of accurate management plans and action points for posterity [21] and efficiency of the MDT meeting. Table 2 summarises the responsibilities of the MDT meeting lead/chair. Ideally the MDT lead/chair is the same person; however, this is not imperative. Due to issues related to job planning and other clinical/research/educational commitments, the MDT chair can be a different physician to the MDT lead. Should this be the case, they should

Table 1 Challenges in setting up a functional MDT

Time commitment

Attendance

Dedication

Leadership

Roles

Processes

Communication

Resources work closely to ensure the smooth running of the service and optimise patient care. An important factor underlying success of the MDT leadership is a common communication code for the lead and the members. In recognition of this need, the ERS has introduced the thoracic oncology HERMES (Harmonising Education in Respiratory Medicine for European Specialists) European Curriculum recommendations for training in thoracic oncology in Europe to ensure successful candidates have the same standards in multidisciplinary thoracic oncology training and can successfully lead their thoracic oncology MDTs regardless of their background specialty [22, 23].

Members' roles and standard operating procedures are of vital importance for a functional MDT (tumour board). Reaching an agreement on these can be challenging when setting up the MDT (tumour board), as MDT members may have different perceptions about their role. Therefore, consultation and final agreement with all MDT members is advised prior to finalising the standard operating procedure. Clear roles are essential to ensure acceptance and engagement from all stakeholders. Achieving successful communication among MDT members, as well as between them and the patients/carers and the GP (general practitioner) can also be challenging. To this effect, dedicated communications skills training is advised for MDT members to ensure improved communication with peers, colleagues, patients and families [24].

Lack of resources (e.g. information technology that projects radiology/nuclear medicine/ pathology results onto a screen) can be a challenge when setting up a MDT (tumour board) meeting as it prevents effective sharing of information among lung cancer specialists and can impact on the quality of the discussion [2]. Information technology (IT) support should be available in real 
Table 2 Responsibilities of the MDT meeting chair/lead

Clear and actionable MDT outcomes (recorded for posterity)

Approval of appropriate cases for discussion and plan for the ones not discussed and diverted to a different pathway

Ensure full case discussion

Ensure equal participation of all specialties and expression of diversity of opinions (where applicable)

Keep meeting on track

Summarise conclusions of discussion for minutes (stage, histology, performance status, management plan)

Determine responsible MDT members for actions decided

MDT meeting outcomes review

Quality assurance

Contingency plans

Service development

time during the MDT (tumour board) meetings and be available on request, especially when teleconferencing is involved.

During the coronavirus disease 2019 (COVID19) pandemic, MDT meetings have become a particular challenge as their established format necessitated face-to-face contact between multiple clinical teams, which could be potent accelerators of viral transmission. As a result, MDT meetings became virtual as a means of reviewing patient care at a physical distance, thereby minimising the risk of infection and maintaining the safety of clinicians. New guidance has been issued on patient prioritisation regarding referral for investigation, treatment and follow-up [25-27].

Table 3 All-inclusive MDT meeting (tumour board) membership

Respiratory physician

Medical oncologist

Radiation (clinical) oncologist

Thoracic surgeon

Interventional pulmonologist

Chest radiologist with interventional expertise

Nuclear medicine

Pathologist

Palliative care

Clinical nurse specialist/nurse support

MDT coordinator

Psychologist (or direct access to them)

Clinical trials coordinator

Nutritionist

Physical occupational therapy

Tracker

Trainees and medical students

\section{MDT membership, roles and responsibilities}

MDT (tumour board) meetings include medical members and AHPs. The medical members of a lung cancer MDT would typically include a respiratory physician, medical oncologist, radiation oncologist (or clinical oncologist), thoracic surgeon, pathologist, dedicated chest radiologist, nuclear medicine physician, and a palliative care physician [3, 7, 28, 29]. Tables 3 and 4 present a list of MDT meeting members and MDT meeting members' (including coordinator's) responsibilities, respectively. MDT meeting members should attend the majority of the meetings and be punctual. When attendance is not possible, there should be arrangements in place for cross cover [28, 29], to ensure all decisions are made with input from all required specialties. Patient discussion and decision making needs to be performed in the presence of at least one team member knowing the patient [28, $29]$ to ensure the optimal decision is taken and the patient's needs are known and are met. MDT meeting members are required to act on the MDT meeting outcomes and ensure all patient related outcomes are completed [28, 29]. MDT meetings ideally should have in attendance more than one representative from each participating specialty to ensure diversity of opinions within the meeting. Administrative support is essential for a functional MDT meeting. The MDT coordinator/secretary plays a pivotal role in an efficient MDT meeting (table 4). In the following sections, we outline the key roles of various specialties during the MDT meeting.

\section{Respiratory physician}

Respiratory physicians have a leading role in the lung cancer MDT meeting as they are involved in the entire spectrum of the lung cancer pathway: prevention, screening, diagnosis, management and follow-up. 
Table 4 MDT meeting members' and coordinator's responsibilities

\begin{tabular}{|c|c|}
\hline Members' responsibilities & Coordinator's responsibilities \\
\hline Meeting attendance and punctuality & $\begin{array}{l}\text { Collate and circulate MDT meeting list prior to the meeting (at least } \\
24 \mathrm{~h} \text { before the meeting) }\end{array}$ \\
\hline Meeting preparation & $\begin{array}{l}\text { Establish links with IT to ensure that IT systems are available and in } \\
\text { use where required }\end{array}$ \\
\hline $\begin{array}{l}\text { Patient discussion and decision making in the presence of } \\
\text { at least one team member knowing the patient }\end{array}$ & MDT meeting room availability and seating arrangements \\
\hline $\begin{array}{l}\text { Minimum one attending member from each specialty to } \\
\text { ensure diversity of opinions }\end{array}$ & $\begin{array}{l}\text { Clear documentation of MDT outcomes by a designated healthcare } \\
\text { professional, member of the MDT meeting (assigned by the chair) }\end{array}$ \\
\hline Action on meeting outcomes & Circulate MDT meeting minutes and action plan \\
\hline Cross cover in case of absence & $\begin{array}{l}\text { Record members' attendance and highlight patterns of non- } \\
\text { attendance to the MDT chair }\end{array}$ \\
\hline Support and participate in quality assurance processes & $\begin{array}{l}\text { Case tracking when a tracker is not available (depending on job } \\
\text { description) }\end{array}$ \\
\hline
\end{tabular}

The respiratory physicians are also the link between the MDT and smoking cessation services. Before or during the MDT, they identify patients that would benefit from smoking cessation and refer them appropriately to ensure they have fast and easy access to the service, for appropriate assessment and management [30].

Depending on the national organisation of primary care, respiratory physicians with a special interest in lung cancer are the first point of referral for patients with suspicion of lung cancer. They lead the diagnostic pathway, and in most European countries (depending on national specialty accreditations) they are at the forefront of treatment alongside medical oncologists [13]. Interventional pulmonologists are also valuable MDT members and need to be physically present in the MDT meeting to identify patients that would benefit from interventional procedures as a treatment/palliative approach/bridge to systemic therapy [31].

\section{Medical oncologist}

Medical oncologists are core members of the MDT meeting and they offer tailored systemic management plans aiming to treat lung cancer, prolong survival and improve quality of life [32].

Considering that cancer is a systemic disease with multiple subtypes, medical oncologists should be able to recognise the safest, least toxic and most cost-efficient drug combination and dosage [32]. They are also skilled to provide input for palliative care in progressive disease and supportive care in cured patients who deal with late complications of cancer therapy [33]. Furthermore, their role contributes to personalised cancer diagnosis by means of molecular analysis techniques and detailed tumour characterisation, which facilitates the administration of targeted therapies against specific cancer types [33]. Their services are also required in the proper management of more demanding cases, such as cancer in the elderly, adolescents or pregnant women. Medical oncologists play a pivotal role in updating the MDT members about available drugs for anticancer treatment, availability and reimbursement of new medications, as well as early access to new clinical trials.

In some institutions, medical oncologists/ respiratory physicians with dedicated training in thoracic oncology, radiation oncologists and molecular pathologists form a separate molecular MDT tumour board that aims to identify patients that would benefit from enrolment in clinical trials providing access to tailored therapies that may be unavailable through national health systems [34].

\section{Radiation (clinical) oncologist}

In the lung cancer MDT meeting, the radiation oncologist contributes to the optimisation of the patient's treatment by complementing the surgeon, the medical oncologist and the respiratory physician, and often has to act and offer a treatment plan to address poor response/disease progression following different preceding treatments [8]. The appropriate selection of radiotherapy technique needs to benefit the patient in terms of efficacy and accuracy and minimise the risk of side-effects. Patients need to be carefully and appropriately selected for IMRT (intensity-modulated radiation therapy), VMAT (Volumetric-modulated arc therapy) or SABR (stereotactic ablative body radiotherapy). The role of the radiation oncologist is to assess all conditions regarding the patient and the tumour, 
to systematically review the need for diagnostic and staging protocols and ensure the best radiation modality is offered. They play a pivotal role in offering radical radiation therapy as a treatment option in patients with early-stage lung cancer who cannot or do not wish to be operated on.

In cases of chemoradiotherapy, the radiation oncologist needs to consider and review the patient's comorbidities and their potential risk to target volume planning. This ensures healthy tissues and vital organs (e.g. heart, liver, spinal column, kidneys) are protected from radiation therapy [8].

\section{Thoracic surgeon}

Clearly, specialisation in thoracic surgery and greater patient volume improve outcome determinants at short-term and long-term evaluations [35]. Thoracic surgeons participating in lung cancer MDT meetings must possess in depth knowledge of thoracic diseases. They should be able to identify candidates for surgical treatment and also highlight those that could benefit from surgical resection but need optimisation of their comorbidities (e.g. optimisation of COPD treatment) and seek appropriate MDT input prior to considering them for surgery. Thoracic surgeons must also possess in-depth knowledge of nonsurgical treatments. This includes innovative medications and radiotherapy techniques that can be applied in inoperable cases. This in-depth knowledge will contribute to the refinement of multimodality treatment approaches in locally advanced lung cancer and will yield important contributions to the development and recruitment of clinical trials in thoracic oncology [36].

\section{Palliative care}

Palliative care representation in the lung cancer MDT ensures early identification of patients that would benefit from early palliative care involvement. Early involvement has shown beneficial results in terms of patients' quality of life and overall clinical outcomes [37]. Palliative care representation in the MDT meeting aims to identify patients that will benefit from specialised input to relieve their cancer/treatment-related symptoms, such as alleviation of pain, breathlessness, and sleep and gastrointestinal disorders. In addition, they can identify patients who are already under palliative care that would benefit from palliative radiotherapy for bone metastasis and facilitate radiation oncology input.

They are also helpful in identifying patients that would benefit from palliative community services and/or hospices and would facilitate this transition to improve patients' experience. These transitions are based on patients' needs and wishes as well as their early involvement in the decision-making of their treatment plan [38].

\section{Radiologists and nuclear medicine}

Dedicated chest radiologists and nuclear medicine physicians play a critical role in lung cancer MDT meetings in terms of accurate staging route to tissue options and radiological follow-up assessments. Meaningful interpretation of radiological modalities, including radiography, computed tomography (CT), magnetic resonance imaging and nuclear medicine modalities (e.g. positron emission tomography CT, bone scintigram), set the basis of the MDT meeting discussion with regards to the optimal management plan $[39,40]$.

\section{Pathologist}

Pathologists' contribution to lung cancer MDT meetings is dynamic and continually evolving. With the development of targeted therapies, the pathologist plays an important role in identifying and performing the most appropriate tests for providing a diagnosis beyond the basic classification into nonsmall or small cell lung cancer, thus allowing access to the most appropriate treatment option [41]. Beyond diagnosis, the pathologists provide reflex testing; thus, allowing biomarker testing to begin as soon as the diagnosis is given, rather than after the patient's first post-biopsy appointment with the oncologist.

On these grounds, the diagnostic small lung/ lymph node biopsy or cytology specimen is a precious resource and the pathologists have become the guardians of these limited samples that aim to maximise their diagnostic yield. Providing timely results to inform the MDT meeting discussions is the result of a long process: specimen adequacy evaluation, selection and application of appropriate processing techniques, selection of suitable slides/ blocks, microdissection to enrich the tumour proportion (where required) and interpretation of the results in a clinical context [41].

\section{Clinical nurse specialist/ oncological nurse}

Clinical nurse specialists/oncology nurses play a crucial role during the MDT meetings as they serve as the patient's advocate throughout their diagnostic, therapeutic and follow-up journey [28, 29]. They ensure patients' wishes and needs are considered and respected during the decisionmaking process in the MDT meeting. They serve as a line of communication between the medical staff and the patient, encouraging frequent contact and emotional support, but also have the responsibility of identifying patients' new needs and facilitate referrals to other healthcare services (e.g. social services) when needed [42].

Their remit focuses also on patient assessment (physical and mental screening), patient and carers'/family education, health needs assessment, 
treatment planning, direct and palliative care and coping with possible side-effects.

Oncological nurses have a concise knowledge of patients' treatment plans and possible adverse reactions in order to provide patients and families accurate information on coping.

\section{Psychologists}

An inclusive MDT should also have psychologists attending the meetings or as a minimum be able to offer direct patient access to psychology services when required [3, 7, 28, 29]. The psychologist plays an important role throughout the patient's pathway, i.e. from diagnostic workup to the of end-of life stage [43]. Specific tools can be applied in each stage of the disease. Facilitating the patient's wellbeing and successful coping with the treatment can be challenging. The psychologist assesses the patient for any pre-existing mental health issues (e.g. personality traits, attachments) and then proceeds with the assessment of psychological distress related to the disease [44]. The interaction between the psychologist and the patient focuses on establishing a trustworthy relationship and meaningful communication that will be able to identify the patient's needs and concerns and arrange a plan to overcome these issues [44]. Psychological assessment, where applicable, should be considered during the MDT meeting to inform the management plan and ensure it is realistic according to the patient's psychological background.

\section{Frequency and number of cases discussed in the MDT meeting}

The frequency of MDT meetings and the number of patients discussed at each meeting varies between institutions. This depends on the size of the centre and the number of referrals, and the robustness of the pre-meeting screening process as well as allocated time for the meeting [28, 29].

Most high-volume centres host at least one MDT meeting per week, while smaller hospitals may not run a regular weekly meeting due to small number of referrals and subsequently patients wait longer for a definitive management plan. Rare tumours (e.g. Pancoast tumours, mesothelioma) raise a similar problem as smaller institutions may not have frequent referrals and professional expertise is limited. In these cases, streamlined care with centralisation in regional specialist MDT meetings is a recommended approach [45]. In rapidly progressive tumours where patient deterioration may be imminent (e.g. small cell lung cancer) there should be "fast track" processes in place to identify these patients and after senior review to refer them to the oncologists for immediate treatment without the need to wait for MDT meeting discussion.
In this case, experienced MDT physicians apply their expertise and special skills without being over reliant on the MDT meeting decision. Urgent decisions need to be taken that are in the patients' best interest. In this group of patients, the risk of clinical deterioration and of missing their window of opportunity for treatment is greater than the benefit of waiting several days for an MDT meeting discussion that will conclude the same plan. As a safety netting, senior MDT member review is required to ensure these patients are appropriately selected and referred for urgent treatment [28, 29, 45].

\section{MDT clinics}

MDT clinics should be linked to the MDT meeting. Ideally, they run "back-to-back", with the initial interdisciplinary assessment taking place in the MDT meeting or the MDT clinic. Based on our experience, we recommend they should run on a "back-to-back" basis, starting with a concise efficient MDT meeting where cases are discussed and then, following a short break, the MDT clinic follows, where all the patients discussed in the meeting will be reviewed in clinic by the relevant specialties as required. Despite accurate minute taking during the MDT [28, 29], it is practically impossible to depict on paper a long conversation for a complex clinical case. However, MDT members attending the meeting and participating in the discussion have a clearer recollection of all aspects of the discussion and of all expressed opinions and they can easier reiterate this during their consultation with the patient when this occurs right after the MDT meeting. This "fresh recollection" can also support a patient's wish for a second opinion, where the healthcare professional will reiterate any second opinions expressed during the MDT prior to the final decision.

Patients' direct access to the MDT clinic enhances multimodality treatment options especially in complex lung cancer cases and gives patients the opportunity to communicate directly with relevant specialties to ensure they comprehend the treatment recommendations and participate in the informed decision-making process [7, 9].

In a pragmatic clinical setting, the philosophy of the MDT clinic can be achieved by obtaining dedicated clinic space for multiple healthcare professionals on the same day and time. The clinic administration team helps patients to navigate from one healthcare professional to the other and achieve consultations from various specialties on the same day. This is time efficient but can sometimes be overwhelming for the patient in terms of volume of information shared. Therefore, it is important that patients are offered relevant patient information leaflets and a written personal plan, they are accompanied by a carer/relative/friend and liaise with their oncology nurse should they have any further questions [7, 9]. 


\section{What is the MDT lung cancer care model meant to achieve?}

The following are considered putative benefits of MDT working [2, 3]:

- Improved consistency, continuity, coordination and cost-effectiveness of care,

- Improved communication between health professionals,

- Improved clinical outcomes,

- Increased recruitment into clinical trials,

- Opportunities to improve audit,

- Increased satisfaction and psychological wellbeing of patients,

- Educational opportunities for health professionals,

- Support from a collegial environment,

- Increased job satisfaction and psychological wellbeing of team members.

Moreover, MDT working is intended to resolve the following issues in cancer care:

- Non-uniform access to specialist care,

- Frequent reporting of deficiencies in cancer services,

- Disjointed referral system,

- Large variations in frequency of individual treatments used, caseload for particular doctors treating cancer, and patient survival.

\section{What is the impact of multidisciplinary care on patients with lung cancer?}

A recent systematic review and meta-analysis assessed the impact of MDT meetings on patient assessment, management and outcomes in oncology settings and concluded that MDT meetings did indeed impact upon cancer patient assessment and management practices [46]. However, there was little evidence indicating that MDT meetings resulted in improvements in clinical outcomes, which is why future research should assess the impact of MDT meetings on patient satisfaction and quality of life, as well as rates of cross-referral between disciplines. Based on the 27 studies included, it was reported that $4-45 \%$ of patients discussed at MDT meetings experienced changes in diagnostic reports following the meeting. Those patients were more likely to receive more accurate and complete pre-operative staging, and neoadjuvant/adjuvant treatment. However, the quality of studies was affected by selection bias and the use of historical cohorts impacted on study quality.

Table 5 summarises studies presenting the impact of multidisciplinary care on lung cancer patients and management practices [47-60].

\section{Impact on diagnosis and staging}

Obtaining a tissue diagnosis is crucial when lung cancer is suspected to ascertain the tumour type and guide further investigation, management, and prognosis [3]. Multidisciplinary care facilitates discussions between the different specialties involved in the diagnostic process [3]. Diagnosis and management of stage III NSCLC is a textbook example of multidisciplinary cancer care. This heterogeneous group needs a coordinated approach to treatment involving surgery, medical and radiation oncology as well as respiratory medicine. A recent analysis of stage III NSCLC patients compared management with and without an MDT meeting and reported that NSCLC patients discussed in MDT meetings had shorter time intervals from first consultation to treatment (20 versus 29 days), higher proportions of mediastinal staging, lower proportions of unsuspected N2 disease, and higher adherence to clinical pathways [60]. The median overall survival appeared longer in the MDT group approaching statistical significance (17 versus 14 months, $p=0.054$ ).

\section{Impact on treatment}

With regards to the effect of MDT care on cancer management, it was shown that multidisciplinary cancer care is more likely to adhere to clinical guidelines and avoid variations in care [3]. In lung cancer, it is established that MDT care increases access to different treatment modalities, including chemo/radiotherapy and surgery, and facilitates recruitment into clinical trials $[3,56]$.

Moreover, patients whose physicians attended weekly discussions at a tumour board were shown to be more likely to undergo curative-intent surgery in stage I and II NSCLC [59]. NSCLC patients with stage I or II disease who did not receive surgery were shown to be more likely to receive radiotherapy if being discussed at the MDT meeting [57]. Timeliness of treatment delivery is one of the key performance indicators of quality care in lung cancer [3]. It has been reported that the multidisciplinary model improves time between diagnosis and initiation of treatment in lung cancer patients [60].

\section{Impact on patient survival}

There is an increasing body of evidence that multidisciplinary cancer care improves patient survival [3]. Even though there seems to be a survival benefit for lung cancer patients if their cases were discussed at an MDT meeting (table 5), these data were derived from mostly observational studies with historical control cohorts.

\section{Impact on patient-centred outcomes: quality of life}

There is evidence of improved quality of life for lung cancer patients after receiving 


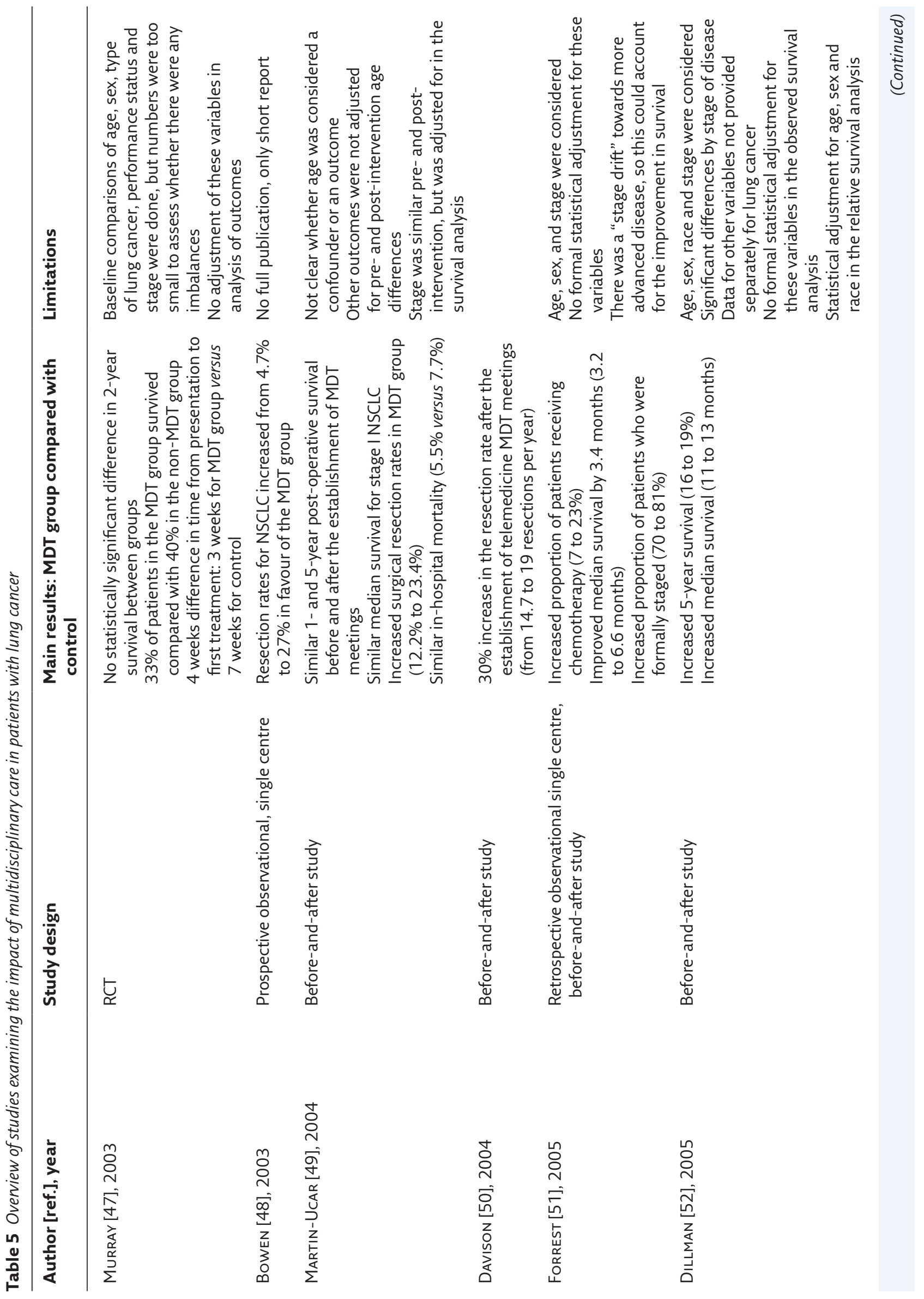




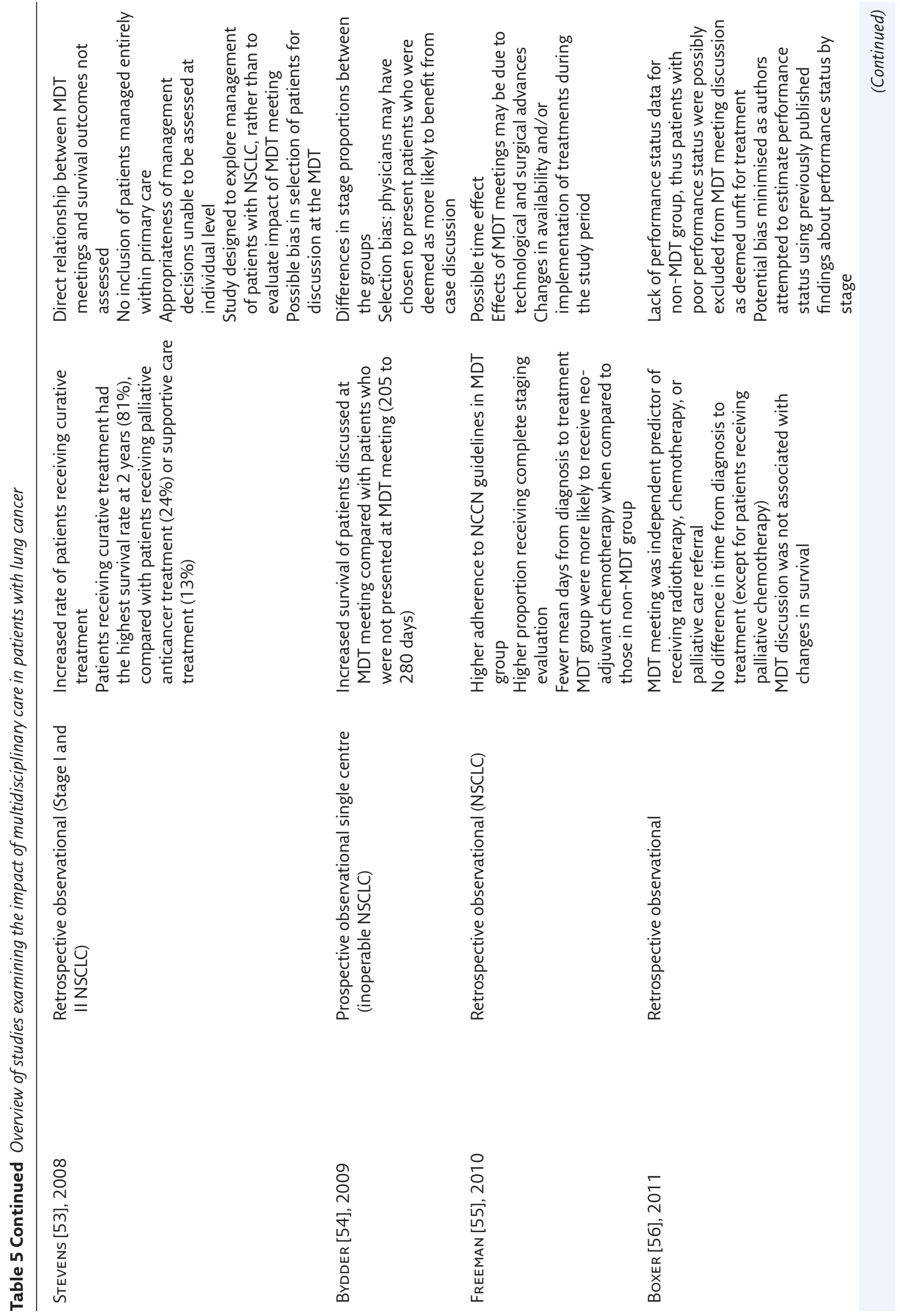


Multidisciplinary care models for patients with lung cancer

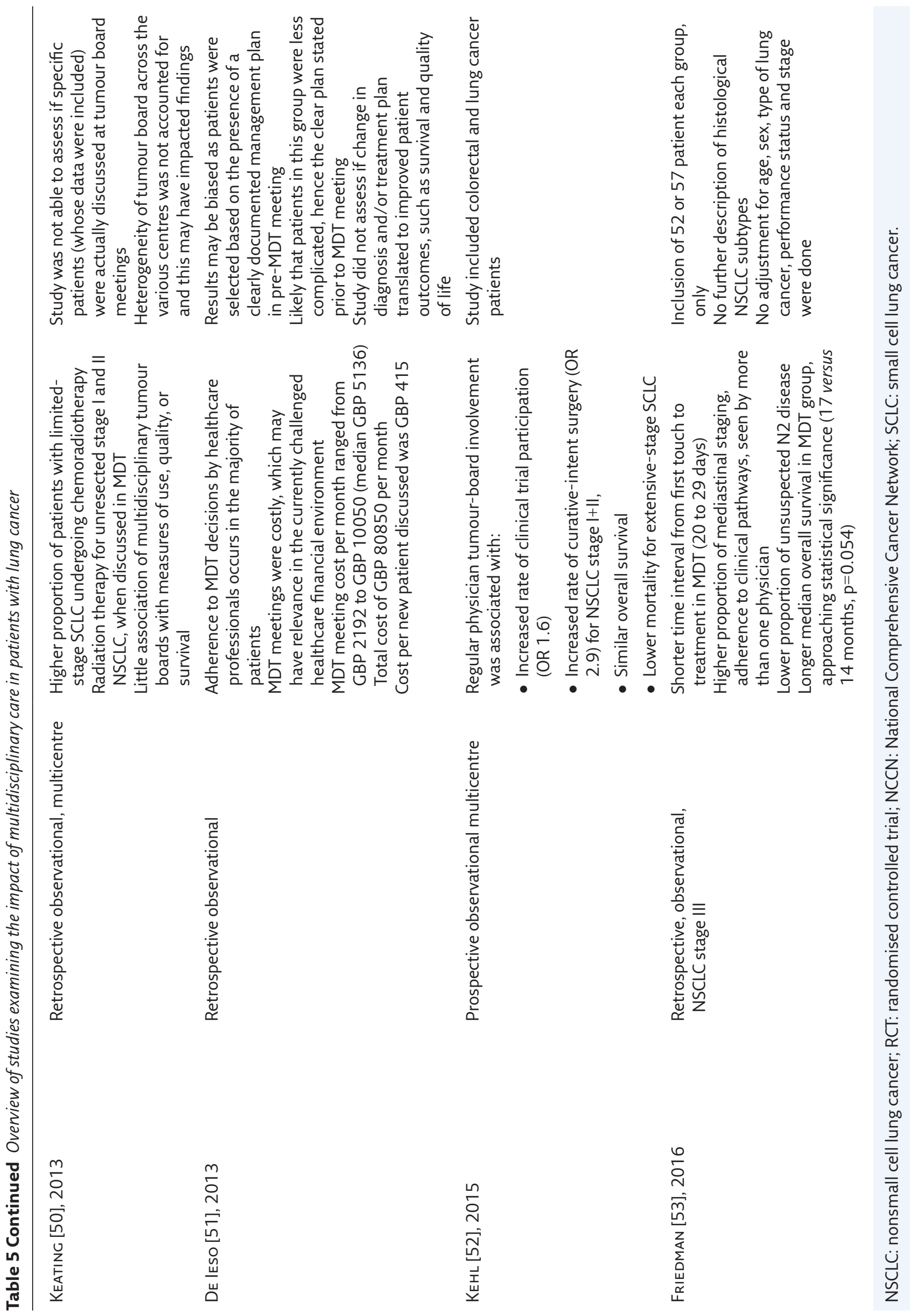




\section{Self-evaluation questions}

1) Which of the following specialties should participate in the lung cancer MDT?
a) Respiratory
b) Medical Student
c) Nuclear Medicine
d) Endocrinology

2) What is/are the responsibilities of the MDT chair?
a) Determine responsible MDT members for actions
b) Circulate MDT meeting minutes and action plan
c) MDT meeting room availability and sitting arrangements
d) Record members' attendance

3) Which of the following is/are challenges when setting up a lung cancer MDT?
a) MDT room arrangement
b) Time commitment
c) Communication
d) Contingency plan

4) Is there robust scientific evidence that MDT meetings improve patient outcomes?
a) Yes
b) No

referred earlier, and subsequently they have a better quality of life and longer survival.

\section{Impact on communication and clinical decision making}

There are a number of important aspects that must occur in a timely fashion to ensure bestquality care in lung cancer [3]. Among others, these include patient-centred treatment decisions, supportive care, follow-up, and surveillance. The coordination of these processes is important for the patient's experience and might pose difficulties if the patient is from a rural or remote area. That is why communication of decisions is crucial to ensure a smooth transition across healthcare services [3]. A multidisciplinary approach facilitates effective communication by ensuring all team members are familiar with the patient's history and are involved in conceptualising the treatment plan [3].

\section{Conclusion}

Multidisciplinary care is the cornerstone of lung cancer care. MDT meetings accompanied by MDT clinics on the same day is the proposed model of care that ensures close interdisciplinary collaboration to meet patients' needs. Clarity on MDT member roles and processes is paramount for an efficient MDT. Overall evidence suggests that multidisciplinary care facilitates the delivery of a high-quality lung cancer service, and that this may result in improved survival, guideline-based treatment, and quality of life for lung cancer patients. However, this has been demonstrated by limited observational data; therefore, more quality evidence is needed to confirm the association between multidisciplinary care and improvements in important lung cancer outcomes.

\footnotetext{
Affiliations

Georgia Hardavella ${ }^{1}$, Armin Frille ${ }^{2,3}$, Christina Theochari' ${ }^{1}$ Elli Keramida ${ }^{1}$, Elena Bellou ${ }^{1}$, Andreas Fotineas ${ }^{4}$, Irma Bracka1, Loukia Pappa', Vaia Zagana ${ }^{5}$, Maria Palamiotou ${ }^{5}$, Panagiotis Demertzis', loannis Karampinis ${ }^{6}$

19th Dept of Respiratory Medicine, "Sotiria” Athens' Chest Diseases Hospital, Athens, Greece. 2Dept of Respiratory Medicine, University of Leipzig, Leipzig, Germany. ${ }^{3}$ Integrated Research and Treatment Center (IFB) Adiposity Diseases, University Medical Center Leipzig, Leipzig, Germany. ${ }^{4}$ Radiation Oncology Dept, IASO Maternity Hospital, Athens, Greece. ${ }^{5}$ Dept of Nursing, "Sotiria” Athens' Chest Diseases Hospital, Athens, Greece. ${ }^{6}$ Dept of Thoracic Surgery, Sismanogleio General Hospital, Athens, Greece.
}

\section{Support statement}

A. Frille was supported by the postdoctoral fellowship "MetaRot program" from the Federal Ministry of Education and Research (BMBF), Germany (FKZ 01E01501, IFB AdiposityDiseases), a research grant from the Mitteldeutsche Gesellschaft für Pneumologie (MDGP) e.V. (2018-MDGP-PA-002), and a junior research grant from the Medical Faculty (Nachwuchsförderprogramm), University of Leipzig. He was stipend of the German Society of Internal Medicine (DGIM, Roland-Müller academy). 
None declared.

\section{References}

1. World Health Organization. Framework for action on interprofessional education and collaborative practice. Geneva, World Health Organization, 2010.www. who.int/hrh/resources/framework_action/en/index.html Date last accessed 26 April, 2020.

2. Cancer Australia. All about multidisciplinary care. www.canceraustralia.gov.au/clinical-best-practice/ multidisciplinary-care/all-about-multidisciplinary-care. Date last accessed: 20 April, 2020.

3. Denton E, Conron M. Improving outcomes in lung cancer: the value of the multidisciplinary health care team. J Multidiscip Healthc 2016; 9: 137-144.

4. Gouveia J, Coleman M, Haward R, et al. Improving cancer control in the European Union: conclusions from the Lisbon round-table under the Portuguese EU presidency, 2007. Eur J Cancer 2008; 44: 1457-1462.

5. Commission on Cancer. Optimal Resources for Cancer Care 2020. American College of Surgeons, 2020. www.facs.org/ Quality-Programs/Cancer/CoC/standards/2020 Date last accessed: 20 April, 2020.

6. Blum TG, Rich A, Baldwin D, et al. The European initiative for quality management in lung cancer care. Eur Respir J 2014; 43: 1254-1277

7. Osarogiagbon RU, Freeman RK, Krasna MJ. Implementing effective and sustainable multidisciplinary clinical thoracic oncology programs. Trans/ Lung Cancer Res 2015; 4: 448-455.

8. Salomaa ER, Sällinen S, Hiekkanen H, et al. Delays in the diagnosis and treatment of lung cancer. Chest 2005; 128 : 2282-2288.

9. Kedia SK, Ward KD, Digney SA, et al. 'One-stop shop': lung cancer patients' and caregivers' perceptions of multidisciplinary care in a community healthcare setting. Transl Lung Cancer Res 2015; 4: 456-464.

10. Osarogiagbon RU, Phelps G, McFarlane J, et al. Causes and consequences of deviation from multidisciplinary care in thoracic oncology. J Thorac Oncol 2011; 6: 510-516.

11. Kedia S, Ward KD, Digney SA, et al. Organizational barriers to receiving quality health care: A qualitative study among lung cancer patients and caregivers. J Clin Oncol 2014; 32: abstr 76.

12. NHS England. MDT Development: Working toward an effective multidisciplinary/multiagency team. NHS England. Version 01. 2015 www.england.nhs.uk/publication/ making-it-happen-multi-disciplinary-team-mdt-working/

13. Gaga M, Powell CA, Schraufnagel DE, et al. An official American Thoracic Society/European Respiratory Society statement: the role of the pulmonologist in the diagnosis and management of lung cancer. Am J Respir Crit Care Med 2013; 188: 503-507.

14. Haas M, Mortensen $M$. The secrets of great teamwork. Harv Bus Rev 2016; 94: 70-76.

15. Royal College of Physicians. Respiratory medicine: Workforce and job planning. www.rcpmedicalcare.org.uk/developingphysicians/specialties/respiratory-medicine/workforce. Date last accessed: 20 April, 2020.

16. Lamb BW, Sevdalis N, Arora S, et al. Teamwork and team decision-making at multidisciplinary cancer conferences: barriers, facilitators, and opportunities for improvement. World J Surg 2011; 35: 1970-1976.

17. Rosell L, Alexandersson N, Hagberg O, et al. Benefits, barriers and opinions on multidisciplinary team meetings: a survey in Swedish cancer care. BMC Health Serv Res 2018; 18: 249.

18. Ruhstaller $\mathrm{T}$, Roe $\mathrm{H}$, Thürlimann $\mathrm{B}$, et al. The multidisciplinary meeting: an indispensable aid to communication between different specialties. Eur J Cancer 2006; 42: 2459-2462.

19. Jalil R, Lamb B, Russ $S$, et al. The cancer multi-disciplinary team from the coordinators' perspective: results from a national survey in the UK. BMC Health Serv Res 2012; 12: 457.
20. Nancarrow S, Booth A, Ariss S, et al. Ten principles of good interdisciplinary team work. Hum Resour Health 2013; 11: 19.

21. Mathioudakis A, Rousalova I, Gagnat AA, et al. How to keep good clinical records. Breathe (Sheff) 2016; 12: 369-373.

22. Gamarra F, Noël JL, Brunelli A, et al. Thoracic oncology HERMES: European curriculum recommendations for training in thoracic oncology. Breathe (Sheff) 2016; 12: 249-255.

23. Hardavella G, Gaga M. Harmonising Education in Thoracic Oncology in Europe; European Recommendations for integrated Training. Pneumon 2016; 29: 203-205.

24. Shaw DJ, Davidson JE, Smilde RI, et al. Multidisciplinary team training to enhance family communication in the ICU. Crit Care Med 2014; 42: 265-271.

25. Sidpra J, Chhabda S, Gaier C, et al. Virtual multidisciplinary team meetings in the age of COVID-19: an effective and pragmatic alternative. Quant Imaging Med Surg 2020; 10 : 1204-1207.

26. European Society for Medical Oncology. ESMO management and treatment adapted recommendations in the COVID-19 era: lung cancer. www.esmo.org/guidelines/cancer-patientmanagement-during-the-covid-19-pandemic/lung-cancerin-the-covid-19-era. Date last accessed: 28 August, 2020.

27. Dingemans AM, Soo RA, Jazieh AR, et al. Treatment guidance for patients with lung cancer during the coronavirus 2019 pandemic. J Thorac Oncol 2020; 15: 1119-1136.

28. National Lung Cancer Working Group. Lung Cancer Multidisciplinary Meeting Toolkit. Wellington, Ministry of Health, 2014.

29. Patkar V, Acosta D, Davidson T, et al. Cancer multidisciplinary team meetings: evidence, challenges, and the role of clinical decision support technology. Int J Breast Cancer 2011; 2011: 831605.

30. Jemal $A$, Ward $E$, Thun $M$. Declining death rates reflect progress against cancer. PLoS One 2010; 5: e9584.

31. Hardavella G, George J. Interventional bronchoscopy in the management of thoracic malignancy. Breathe (Sheff) 2015; 11: 202-212.

32. Popescu RA, Schaefer R, Califano R, et al. The current and future role of the medical oncologist in the professional care for cancer patients: a position paper by the European Society for Medical Oncology (ESMO). Ann Oncol 2014; 25: 9-15.

33. Cherny NI, Catane R, Kosmidis P. ESMO takes a stand on supportive and palliative care. Ann Oncol 2003; 14: 1335-1337.

34. Basse C, Morel C, Alt M, et al. Relevance of a molecular tumour board (MTB) for patients' enrolment in clinical trials: experience of the Institut Curie. ESMO Open 2018; 3 : e000339.

35. Falcoz PE, Puyraveau M, Rivera CM, et al. The impact of hospital and surgeon volume on the 30-day mortality of lung cancer surgery: A nation-based reappraisal. J Thorac Cardiovasc Surg 2014; 148: 841-848.

36. Massard G, Antonoff MB, Noel JL, et al. Transatlantic editorial: Thoracic surgeons need recognition of competence in thoracic oncology. J Thorac Cardiovasc Surg 2017; 154: 1387-1392.

37. Temel JS, Greer JA, Muzikansky A, et al. Early palliative care for patients with metastatic non-small-cell lung cancer. N EnglJ Med 2010; 363: 733-742.

38. Spronk I, Meijers MC, Heins MJ, et al. Availability and effectiveness of decision aids for supporting shared decision making in patients with advanced colorectal and lung cancer: Results from a systematic review. Eur J Cancer Care (Engl) 2019; 28: e13079.

39. Prabhakar CN, Fong KM, Peake MD, et al. The effectiveness of lung cancer MDT and the role of respiratory physicians. Respirology 2015; 20: 884-888.

\section{Suggested answers}

1. a, b, c.

2. a.

3. a-d.

4. b. 
40. Lim E, Baldwin D, Beckles M, et al. Guidelines on the radical management of patients with lung cancer. Thorax 2010; 65 1-27.

41. Davidson MR, Gazdar AF, Clarke BE. The pivotal role of pathology in the management of lung cancer. J Thorac Dis 2013; 5 Suppl 5: S463-78.17

42. McPhillips D, Evans R, Ryan D, et al. The role of a nurse specialist in a modern lung-cancer service. BrJ Nurs 2015 24: S21-S27.

43. Baider L, Surbone A. Universality of aging: family caregivers for elderly cancer patients. Front Psychol 2014 Jul 15; 5 744.

44. Castelli L, Binaschi L, Caldera P, et al. Fast screening of depression in cancer patients: the effectiveness of the HADS EurJ Cancer Care (Engl) 2011; 20: 528-533.

45. Postmus PE. Longkanker: centraliseren voor multidisciplinaire behandeling. Ned Tijdschr Geneeskd 2007; 151: 1382-1384.

46. Pillay $B$, Wootten AC, Crowe $\mathrm{H}$, et al. The impact of multidisciplinary team meetings on patient assessment management and outcomes in oncology settings: A systematic review of the literature. Cancer Treat Rev 2016; 42: 56-72.

47. Murray PV, O’Brien ME, Sayer R, et al. The pathway study: results of a pilot feasibility study in patients suspected of having lung carcinoma investigated in a conventional chest clinic setting compared to a centralised two-stop pathway. Lung Cancer 2003; 42: 283-290.

48. Bowen EF, Anderson JR, Roddie ME. Improving surgical resection rates in lung cancer without a two stop service. Thorax 2003; 58: 368.

49. Martin-Ucar AE, Waller DA, Atkins JL, et al. The beneficial effects of specialist thoracic surgery on the resection rate for non-small-cell lung cancer. Lung Cancer 2004; 46: 227-232.

50. Davison AG, Eraut CD, Haque AS, et al. Telemedicine for multidisciplinary lung cancer meetings. J Telemed Telecare 2004; 10: 140-143.
51. Forrest LM, McMillan DC, McArdle CS, et al. An evaluation of the impact of a multidisciplinary team, in a single centre, on treatment and survival in patients with inoperable non-smallcell lung cancer. BrJ Cancer 2005; 93: 977-978.

52. Dillman RO, Chico SD. Cancer patient survival improvement is correlated with the opening of a community cancer center: comparisons with intramural and extramural benchmarks. J Oncol Pract 2005; 1: 84-92.

53. Stevens W, Stevens G, Kolbe J, et al. Management of stages I and II non-small-cell lung cancer in a New Zealand study: divergence from international practice and recommendations. Intern Med J 2008; 38: 758-768.

54. Bydder S, Nowak A, Marion K, et al. The impact of case discussion at a multidisciplinary team meeting on the treatment and survival of patients with inoperable non-small cell lung cancer. Intern Med J 2009; 39: 838-841.

55. Freeman RK, Van Woerkom JM, Vyverberg A et al. The effect of a multidisciplinary thoracic malignancy conference on the treatment of patients with lung cancer. Eur J Cardiothorac Surg 2010; 38: 1-5.

56. Boxer MM, Vinod SK, Shafiq J, et al. Do multidisciplinary team meetings make a difference in the management of lung cancer? Cancer 2011; 117: 5112-5120.

57. Keating NL, Landrum MB, Lamont EB, et al. Tumor boards and the quality of cancer care. J Natl Cancer Inst 2013; 105 : 113-121.

58. De leso PB, Coward JI, Letsa I, et al. A study of the decision outcomes and financial costs of multidisciplinary team meetings (MDMs) in oncology. Br J Cancer 2013; 109: 2295-2300

59. Kehl KL, Landrum MB, Kahn KL, et al. Tumor board participation among physicians caring for patients with lung or colorectal cancer. J Oncol Pract 2015; 11: e267-e278.

60. Friedman EL, Kruklitis RJ, Patson BJ, et al. Effectiveness of a thoracic multidisciplinary clinic in the treatment of stage III nonsmall-cell lung cancer. J Multidiscip Healthc 2016; 9: 267-274. 